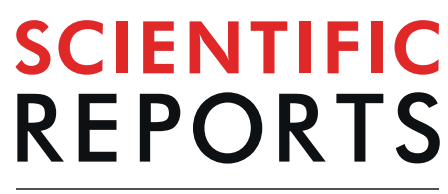

natureresearch

\title{
Artificial liver support system therapy in acute-on-chronic hepatitis B liver failure: Classification and regression tree analysis
}

\author{
Kaizhou Huang ${ }^{1,3}$, Feiyang $\mathrm{Ji}^{1,3}$, Zhongyang $\mathrm{Xie}^{1,3}$, Daxian $\mathrm{Wu}^{1}$, Xiaowei $\mathrm{Xu}^{1}$, Hainv $\mathrm{Gao}^{2}$, \\ Xiaoxi Ouyang ${ }^{1}$, Lanlan Xiao ${ }^{1}$, Menghao Zhou ${ }^{1}$, Danhua Zhu ${ }^{1}$ \& Lanjuan $\mathrm{Li}^{1 *}$
}

Artificial liver support systems (ALSS) are widely used to treat patients with hepatitis B virus-related acute-on-chronic liver failure (HBV-ACLF). The aims of the present study were to investigate the subgroups of patients with HBV-ACLF who may benefit from ALSS therapy, and the relevant patientspecific factors. 489 ALSS-treated HBV-ACLF patients were enrolled, and served as derivation and validation cohorts for classification and regression tree (CART) analysis. CART analysis identified three factors prognostic of survival: hepatic encephalopathy (HE), prothrombin time (PT), and total bilirubin (TBil) level; and two distinct risk groups: low (28-day mortality 10.2-39.5\%) and high risk (63.8-91.1\%). The CART model showed that patients lacking HE and with a PT $\leq 27.8 \mathrm{~s}$ and a TBil level $\leq 455 \mu \mathrm{mol} / \mathrm{L}$ experienced less 28-day mortality after ALSS therapy. For HBV-ACLF patients with HE and a PT $>27.8 \mathrm{~s}$, mortality remained high after such therapy. Patients lacking HE with a PT $\leq 27.8 \mathrm{~s}$ and TBil level $\leq 455 \mu \mathrm{mol} / \mathrm{L}$ may benefit markedly from ALSS therapy. For HBV-ACLF patients at high risk, unnecessary ALSS therapy should be avoided. The CART model is a novel user-friendly tool for screening HBV-ACLF patient eligibility for ALSS therapy, and will aid clinicians via ACLF risk stratification and therapeutic guidance.

Acute-on-chronic liver failure (ACLF) is a complicated syndrome that can cause rapid deterioration in patients with chronic liver disease, associated with high-level mortality ${ }^{1}$. Several large, prospective multicentre studies have shown that patients with ACLF have extremely bad prognoses; the 28 -day mortality rate ranges from $30 \%$ to $90 \%{ }^{2-4}$. In Asian, Hepatitis B virus (HBV) infection accounts for the majority of ACLF ${ }^{3}$. Recently, new diagnostic criteria for HBV-ACLF and a prognostic scoring system were developed in a prospective work conducted by the Chinese Group on the Study of Severe Hepatitis B-ACLF (COSSH-ACLF) (1322 patients in 13 liver centres were studied $)^{4}$. Liver transplantation (LT) effectively treats HBV-ACLF patients who respond poorly to standard treatment, but is limited by organ scarcity. Over the past three decades, artificial liver support systems (ALSS) have been employed to treat liver failure. Previous studies found that ALSS improved short-term survival in those with acute-on-chronic liver failure ${ }^{5,6}$. Some studies, including a prospective controlled study, found that ALSS was safe, well tolerated, and a useful bridge to LT in patients with $\mathrm{ACLF}^{7-9}$. However, another study found that HBV-ACLF patients with lower Model for End-stage Liver Disease scores (MELDs) enjoyed significantly better outcomes than did those with higher MELDs ${ }^{10}$. Other studies suggested that ALSS afforded survival benefits in specific groups ${ }^{11,12}$. Thus, subgroups of patients who can benefit from HBV-ACLF, and factors affecting survival, must be identified. To guide and optimise targeted therapy for HBV-ACLF patients, a practical, accurate decision-making

\footnotetext{
${ }^{1}$ State Key Laboratory for Diagnosis and Treatment of Infectious Diseases, National Clinical Research Center for Infectious Diseases, Collaborative Innovation Center for Diagnosis and Treatment of Infectious Diseases, The First Affiliated Hospital of Zhejiang University, College of Medicine, Zhejiang University, Hangzhou, Zhejiang Province, China. ${ }^{2}$ Shulan Hangzhou Hospital, Shulan Health, Hangzhou, Zhejiang Province, China. ${ }^{3}$ These authors contributed equally: Kaizhou Huang, Feiyang Ji and Zhongyang Xie. *email: ljli@zju.edu.cn
} 


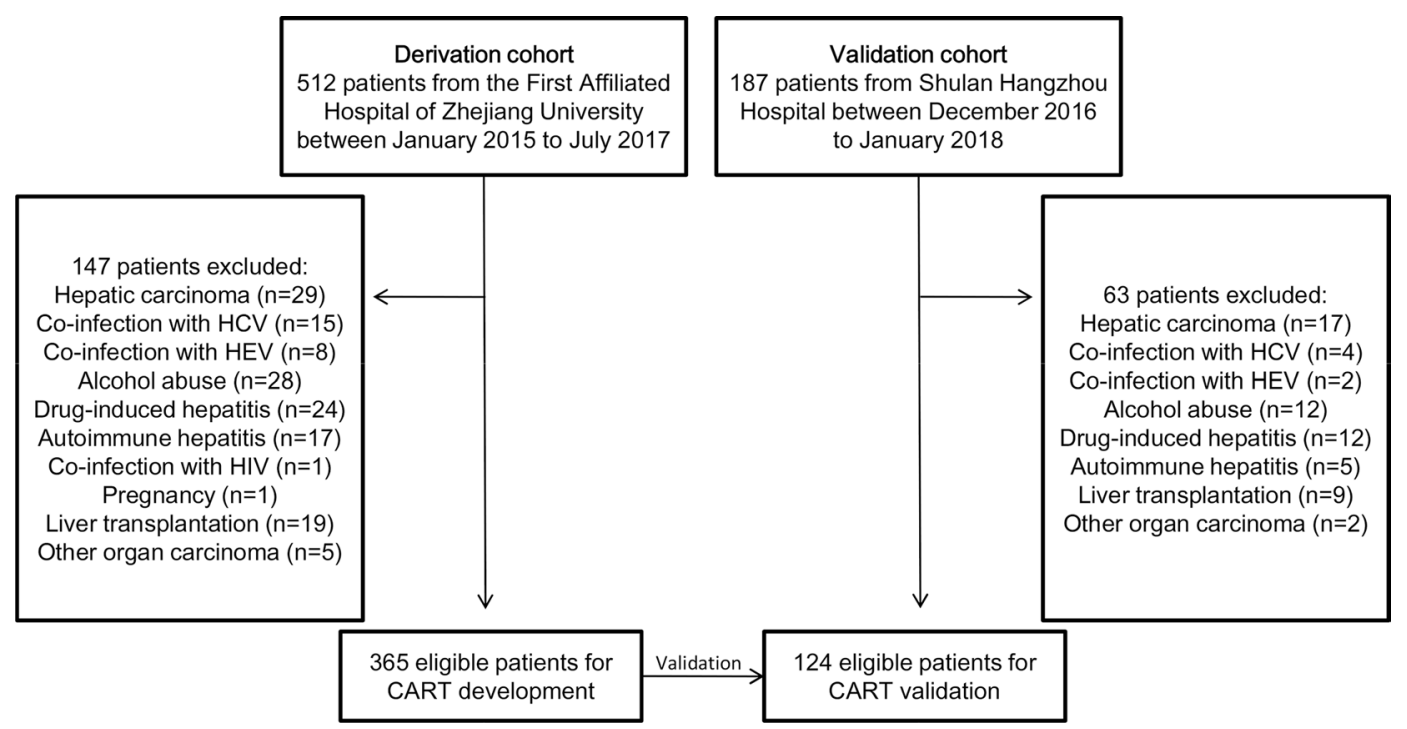

Figure 1. A flow diagram of study participants included in the study.

tool is urgently needed to help physicians evaluate risks and decide whether to initiate ALSS therapy or to prefer conservative treatment.

Unlike multivariable logistic regression, classification and regression tree (CART) analysis is a non-parametric algorithm based on recursive partitioning. CART analysis separates all values using a decision tree featuring progressive binary splits. Several splits serve as predictors identifying patients at different degrees of risk. Given its convenience and clinical utility, CART analysis has been used to develop predictive models aiding clinical decision-making in various medical fields ${ }^{13-15}$. To help clinicians identify and screen patients eligible for ALSS therapy, we developed an accurate, user-friendly, bedside prognostic model employing CART analysis. We compared the accuracy of our model in term of predicting 28-day mortality to that of a new Z logistic regression model (LRM-Z) and certain older prognostic models, including the model for end-stage liver disease (MELD), integrated model for end-stage liver disease (iMELD), Chronic Liver Failure Consortium acute-on-chronic liver failure (CLIF-C ACLF) score and Chinese group on the Study of Severe Hepatitis B-acute-on-chronic liver failure (COSSH-ACLF) score.

\section{Results}

Baseline characteristics of the derivation and validation cohorts. A total of 699 hospitalised HBVACLF patients were initially screened and enrolled; 365 patients in the derivation cohort and 124 in the validation cohort were ultimately included (Fig. 1). The baseline characteristics of both cohorts of patients are listed in Table 1 and Supplementary Table 1. Most patients in the derivation cohort were male and had lower rates of liver cirrhosis $(P<0.05)$. The derivation cohort had lower ALB, glucose, BUN and NH3 levels, and a lower WBC count, than the validation cohort, which in turn had lower D-dimer, ALP, Hb and Plt levels than the deviation cohort (all $P<0.05$ ). No significant differences in terms of model scores or mortality were evident between the two cohorts. The characteristics of the derivation and validation cohorts stratified by 28-day mortality are shown in Table 2. The cohorts were similar in terms of the variables significantly influencing survival, i.e. the HE proportion; WBC count; the levels of ALT, TBil, INR, PT, fibrinogen, D-dimer, Hb and NH3; and the scores on all systems tested (all $P<0.05$ ). No significant differences were found between ALSS sessions and mortality in both two cohorts (Supplementary Table 2).

CART and LRM-Z analysis in the derivation cohort. In CART analysis, HE status served as the initial variable. After HE separation, a PT of $27.8 \mathrm{~s}$ was chosen as the second split variable in patients lacking HE. When the $\mathrm{PT} \leq 27.8 \mathrm{~s}$, the next best predictor was the TBil level, at an optimal cut-off of $455 \mu \mathrm{mol} / \mathrm{L}$. No additional node afforded any increment in risk discrimination. Therefore, four subgroups of patients, differing significantly in terms of 28-day mortality, were generated by three predictive variables identified via CART analysis: subgroup 1 (patients with HE); subgroup 2 (patients lacking HE but with a PT $>27.8$ s); subgroup 3 (patients lacking HE and with a PT $\leq 27.8$ s, but a TBil level $>455 \mu \mathrm{mol} / \mathrm{L}$ ); and subgroup 4 (patients lacking HE with a PT $\leq 27.8$ s, and a TBil level $\leq 455 \mu \mathrm{mol} / \mathrm{L}$ ) (Fig. 2). Subgroups 1 and 2 were combined to form a high-risk group, with mortality rates ranging from 63.7 to $87.2 \%$, whereas subgroups 3 and 4 were combined to form a low-risk group, with mortality rates ranging from 10.2 to $39.5 \%$. HBV-ACLF patients in the high-risk group exhibited a 9.883-fold (95\% CI: 6.002-16.275-fold, $P<0.001)$ increased in 28-day mortality (compared to those in the low-risk group) after ALSS-treatment (Table 3). Detailed descriptions of LRM-Z analysis are given in the Supplemental Results and Supplementary Table 3.

Validation and comparison. CART analysis was validated for its efficiency of risk stratification efficiency in an independent validation cohort containing 124 subjects. Using the flow chart of the classification tree, each patient was allocated to a subgroup (Supplementary Fig. 1). All patients were also stratified into lowand high-risk groups. Compared to those in the low-risk group, patients in the high-risk group exhibited an 


\begin{tabular}{|c|c|c|c|}
\hline Variable & $\begin{array}{l}\text { Derivation Cohort } \\
(\mathrm{n}=365)\end{array}$ & $\begin{array}{l}\text { Validation Cohort } \\
(\mathrm{n}=124)\end{array}$ & $P$-value \\
\hline \multicolumn{4}{|l|}{ Clinical parameters } \\
\hline Age (years) & $46.52 \pm 11.38$ & $45.31 \pm 11.97$ & 0.314 \\
\hline Male Gender (\%) & $325(89.04 \%)$ & $99(79.84 \%)$ & 0.009 \\
\hline HTN (\%) & $43(11.78 \%)$ & $17(13.71 \%)$ & 0.572 \\
\hline $\mathrm{DM}(\%)$ & $28(7.67 \%)$ & $16(12.90 \%)$ & 0.101 \\
\hline Liver Cirrhosis (\%) & $157(43.01 \%)$ & $77(62.10 \%)$ & $<0.001$ \\
\hline Ascites (\%) & $166(45.48 \%)$ & $68(54.84 \%)$ & 0.071 \\
\hline Hepatic Encephalopathy (\%) & $47(12.88 \%)$ & $16(12.90 \%)$ & 0.996 \\
\hline Hepatic Encephalopathy I-II (\%) & $27(7.40 \%)$ & $9(7.26 \%)$ & \\
\hline Hepatic Encephalopathy III-IV (\%) & $20(5.48 \%)$ & $7(5.65 \%)$ & \\
\hline Infection (\%) & $59(16.16 \%)$ & $29(23.39 \%)$ & 0.070 \\
\hline Gastrointestinal hemorrhage (\%) & $10(2.74 \%)$ & $8(6.45 \%)$ & 0.580 \\
\hline 28 days-Mortality & $140(38.36 \%)$ & $51(41.13 \%)$ & 0.585 \\
\hline \multicolumn{4}{|l|}{ Laboratory parameters } \\
\hline $\operatorname{ALT}(\mathrm{U} / \mathrm{L})$ & $182.00(91.50,371.50)$ & $155.5(59.25,445.75)$ & 0.376 \\
\hline $\operatorname{AST}(\mathrm{U} / \mathrm{L})$ & $138.00(91.00,252.00)$ & $143.50(65.25,404.50)$ & 0.782 \\
\hline $\mathrm{TBil}(\mu \mathrm{mol} / \mathrm{L})$ & $424.87 \pm 123.14$ & $402.11 \pm 132.01$ & 0.082 \\
\hline $\operatorname{ALB}(g / L)$ & $32.19 \pm 6.40$ & $33.75 \pm 4.48$ & 0.012 \\
\hline GGT (U/L) & $70.00(50.50,92.50)$ & $70.00(43.50,119.00)$ & 0.706 \\
\hline Glucose (mmol/L) & $3.96(3.27,4.72)$ & $6.05(4.27,8.70)$ & $<0.001$ \\
\hline INR & $2.11(1.76,2.78)$ & $2.28(1.80,3.25)$ & 0.054 \\
\hline PT (s) & $23.4(19.8,30.7)$ & $23.35(20.03,35.25)$ & 0.209 \\
\hline Fibrinogen $(\mathrm{g} / \mathrm{L})$ & $1.21(0.96,1.55)$ & $1.29(0.96,1.71)$ & 0.294 \\
\hline $\mathrm{D}$-dimer $(\mu \mathrm{g} / \mathrm{L})$ & $\begin{array}{l}2279.00(1120.00 \\
3690.00)\end{array}$ & $\begin{array}{l}1318.00(562.00 \\
3004.50)\end{array}$ & $<0.001$ \\
\hline $\operatorname{ALP}(\mathrm{U} / \mathrm{L})$ & $\begin{array}{l}130.00(108.50 \\
161.00)\end{array}$ & $118.50(95.00,153.75)$ & 0.006 \\
\hline $\mathrm{WBC}\left(10^{9} / \mathrm{L}\right)$ & $8.04 \pm 4.13$ & $9.10 \pm 5.40$ & 0.023 \\
\hline $\mathrm{Hb}(\mathrm{g} / \mathrm{L})$ & $126.04 \pm 19.73$ & $115.24 \pm 28.43$ & $<0.001$ \\
\hline Plt $\left(10^{\wedge} 9 / \mathrm{L}\right)$ & $108.18 \pm 48.80$ & $95.73 \pm 53.82$ & 0.017 \\
\hline Serum Sodium (mmol/L) & $136.79 \pm 4.53$ & $137.29 \pm 9.09$ & 0.428 \\
\hline Creatinine $(\mu \mathrm{mol} / \mathrm{L})$ & $67.00(58.50,80.00)$ & $63.50(49.00,95.00)$ & 0.071 \\
\hline $\mathrm{GFR}(\mathrm{ml} / \mathrm{min})$ & $106.83(92.79,116.36)$ & $\begin{array}{l}111.58(100.92, \\
116.09)\end{array}$ & 0.090 \\
\hline $\mathrm{BUN}(\mathrm{mmol} / \mathrm{L})$ & $4.30(3.30,5.80)$ & $5.00(3.40,8.48)$ & 0.003 \\
\hline $\mathrm{NH} 3(\mu \mathrm{mol} / \mathrm{L})$ & $67.00(50.00,91.50)$ & $80.70(51.00,118.00)$ & 0.002 \\
\hline HBV-DNA $(\log 10, \mathrm{IU} / \mathrm{ml})$ & $5.04 \pm 2.23$ & $4.61 \pm 1.81$ & 0.055 \\
\hline \multicolumn{4}{|l|}{ Scoring system } \\
\hline MELD score & $25.40 \pm 5.55$ & $26.17 \pm 7.54$ & 0.297 \\
\hline iMELD score & $43.60 \pm 7.59$ & $43.66 \pm 10.69$ & 0.953 \\
\hline CLIF-C ACLF score & $41.17 \pm 7.00$ & $41.89 \pm 7.39$ & 0.327 \\
\hline COSSH-ACLF score & $6.34(5.83,6.96)$ & $6.59(5.91,7.52)$ & 0.056 \\
\hline
\end{tabular}

Table 1. Baseline characteristics of derivation and validation cohorts. Abbreviations: HTN, hypertension; DM, diabetes mellitus; ALT, alanine aminotransferase; AST, aspartate aminotransferase; TBil, total bilirubin; ALB, albumin; GGT, gamma-glutamyl transpeptidase; INR, internationalized normal ration; PT, prothrombin time; ALP, alkaline phosphatase; WBC, white blood cell; Hb, hemoglobin; Plt, platelet; GFR, glomerular filtration rate; $\mathrm{BUN}$, urea nitrogen; HBV-DNA, hepatitis B virus- deoxyribonucleic acid; MELD, Model for End-stage Liver Disease; iMELD, integrated model for end-stage liver disease; CLIF-C ACLF, Chronic Liver Failure Consortium acute-on-chronic liver failure; COSSH-ACLF, Chinese group on the Study of Severe Hepatitis B-acute-on-chronic liver failure.

8.485-fold (95\% CI: 3.726-19.202-fold, $P<0.001)$ increase in 28 -day mortality, similar to what was found in the derivation cohort (Table 3 ).

The predictive power in terms of 28-day mortality of ALSS-treated HBV-ACLF patients was compared among the CART, LRM-Z and some earlier prognostic models (Fig. 3). In the derivation cohort (Fig. 3A), CART analysis afforded high performance, with an auROC of 0.824 (95\% CI: 0.781-0.862). LRM-Z and COSSH-ACLF afforded similar accuracies, with auROCs of 0.842 (95\% CI: $0.800-0.878, P=0.1919)$ and 0.800 (95\% CI: 0.755$0.840, P=0.1746)$, respectively. The MELD, iMELD and CLIF-C ACLF had lower auROCs than the CART, 0.727 
(95\% CI: 0.678-0.772, $P=0.0001$ ), 0.675 (95\% CI: 0.625-0.723, $P<0.0001$ ) and 0.742 (95\% CI: 0.694-0.786, $P=0.0011$ ) respectively (Table 4 , Supplementary Table 4 ).

In validation (Fig. 3B), CART analysis featured the highest auROC, 0.820 (95\% CI: 0.741-0.883), and afforded better performance with higher statistical significance than MELD $(0.686,95 \% \mathrm{CI}: 0.597-0.766, P=0.0070)$ and iMELD $(0.685,95 \%$ CI: 0.596-0.766, $P=0.0152)$. Although statistical significance was not attained, LRM-Z, CLIF-C ACLF and COSSH-ACLF all had lower auROCs [0.807 (95\% CI: 0.727-0.873, $P=0.6621), 0.738$ (95\% CI: $0.651-0.812, P=0.0985)$, and 0.810 (95\% CI: $0.730-0.875, P=0.7523)$, respectively] than CART analysis.

\section{Discussion}

ACLF is one of the most intractable clinical problems worldwide, characterised by severe hepatic abnormalities and rapid disease progression ${ }^{16}$. The short-term mortality rate of HBV-ACLF is extremely high; it is essential to stratify patients by their current condition and possible prognosis to select appropriate treatment strategies ${ }^{17}$. Liver transplantation is optimal, but is compromised by donor organ scarcity and the need to select patients carefully ${ }^{18,19}$. ALSS therapies have been considered useful to replace liver function, affording an opportunity for hepatic recovery or stabilising the clinical status prior to transplantation ${ }^{20}$. However, the optimal timing of ALSS treatment and the target population remain remains unclear. Clinicians are in urgent need of a better method to correctly identify patients that would benefit from such treatment, to avoid unnecessary clinical burdens ${ }^{21}$. It is essential to screen patients with ACLF and in terms of factors that would allow them to benefit from ALSS therapy. Here, we established and validated a CART approach toward analysis and identification of predictive factors in subgroups of patients with HBV-ACLF who would benefit from ALSS therapy.

$\mathrm{HE}$ is the most common complication of HBV-ACLF. Previous studies reported that HE in hospitalised ACLF patients was associated with a high mortality rate ${ }^{22}$. Another study indicated that HE obviously affected the clinical prognosis of such patients ${ }^{23}$. Here, LRM-Z confirmed that HE was independently prognostic of 28-day mortality. In CART analysis, HE was the first variable split. HBV-ACLF patients with HE were allocated to subgroup 1 ; the 28 -day mortalities were 87.2 and $87.5 \%$ in the derivation and validation cohorts, respectively. The high 28-day mortality rate of subgroup 1 suggests that HBV-ACLF patients with HE might have difficulty in earning benefits from ALSS therapy. It is generally accepted that hyperbilirubinaemia and coagulopathy are the two most prominent features of liver failure, as indicated by both official criteria and studies on ACLF diagnosis and prognosis worldwide ${ }^{2,3,24,25}$. PT and the TBil level were positively associated with mortality risk in both CART and LRM-Z analyses. PT was the second variable split in CART analysis and was also an independent prognostic factor in the LRM-Z model, suggesting a positive correlation between PT and ACLF mortality, consistent with data from previous studies ${ }^{26,27}$. It is also well received for TBil level to be the third split variable. Our results are in line with previous studies reporting that the TBil level was independently prognostic of ACLF ${ }^{28,29}$. CART analysis also stratified subjects into low- and high-risk groups that exhibited significant differences. Patients in the low-risk group exhibited lower than usual 28-day mortality compared to most patients with HBV-ACLF ${ }^{4}$, strongly indicating that ALSS therapy may improve overall survival in such patients. However, patients in the high-risk group may be difficult to earn benefits from ALSS therapy; LT is required as soon as possible.

Compared to traditional multivariate models, CART analysis has unique advantages. First, CART analysis can process high-dimensional data (even highly skewed data) when the sample size is low ${ }^{30}$. CART analysis can calculate probabilities and impurities using the non-missing values; missed values are ignored ${ }^{31}$. From our results, CART is very comparable to models using logistic regression in terms of predictive value of mortality. However, models constructed with the aid of various logistic regression coefficients are very complex, and clinical utility is compromised. Given its simple classification parameters and cut-off values, the CART model is simple and user-friendly in the hands of clinicians. Third, the CART analysis stratified patients into low- and high-risk groups exhibiting significant differences. Thus, the model facilitates subgroup/risk stratification in a manner similar to how clinicians make decisions, which may improve the management of hospitalised patients with HBV-ACLF. Patients at lower risk can be reassured that ALSS therapy will play a positive and active role in their treatment programs, but unnecessary ALSS therapy should be avoided, and LT prioritised in patients at higher risk.

Our study had certain limitations. First, potential confounders in small data samples may cause the significance of included risk factors to be overestimated, thus influencing the actual risk, which may explain why the prognostic factors identified by the CART model and LRM-Z differed ${ }^{32}$. Further work with a larger population is needed. Second, the CART was built in a single centre and validated in another centre, using homogeneous data. The multicentre adaptability and feasibility of CART requires further verification. Third, only HBV-infected patients were included. To generalise its use, the CART model requires further validation in patients with ACLF of diverse aetiologies. Thus, multicentre, prospective studies with larger patient populations are needed to further verify the applicability of our model.

However, the CART model is a novel, validated, user-friendly bedside tool that can screen HBV-ACLF patients in terms of eligibility for ALSS therapy. HBV-ACLF patients lacking HE and with a PT $\leq 27.8$ s may benefit from such therapy. In this group, the benefit may be more pronounced when the TBil level $\leq 455 \mu \mathrm{mol} / \mathrm{L}$. The CART model helps physicians correctly identify patients at lower risk (facilitating appropriate ALSS use as part of a treatment program), and to prioritise liver transplantation for patients at higher risk.

\section{Methods}

Patients. Patients were screened at two different medical centres operating identical medical record systems. Medical data prior to ALSS therapy were collected from patient records, and derivation and validation cohorts were defined (derivation cohort: the First Affiliated Hospital of Zhejiang University [patients treated between January 2015 and July 2017]; validation cohort: Shulan Hangzhou Hospital [patients treated between December 2016 and January 2018]). The study protocol conformed to the ethical guidelines of the 1975 Declaration of 


\begin{tabular}{|c|c|c|c|c|c|c|}
\hline \multirow[b]{2}{*}{ Variable } & \multicolumn{3}{|l|}{ Derivation Cohort $(n=365)$} & \multicolumn{3}{|c|}{ Validation Cohort $(n=124)$} \\
\hline & Survival $(\mathbf{n}=\mathbf{2 2 5})$ & Death $(n=140)$ & P-value & Survival $(n=73)$ & Death $(n=51)$ & $P$-value \\
\hline \multicolumn{7}{|l|}{ Clinical parameters } \\
\hline Age (years) & $46.48 \pm 11.48$ & $46.57 \pm 11.27$ & 0.940 & $44.04 \pm 12.57$ & $47.12 \pm 10.91$ & 0.160 \\
\hline Male Gender (\%) & $199(88.44 \%)$ & $126(88.57 \%)$ & 0.644 & $56(76.71 \%)$ & $43(84.31 \%)$ & 0.299 \\
\hline HTN (\%) & $27(12.00 \%)$ & $16(11.43 \%)$ & 0.869 & $8(10.96 \%)$ & $9(17.65 \%)$ & 0.287 \\
\hline $\mathrm{DM}(\%)$ & $17(7.56 \%)$ & $11(7.86 \%)$ & 0.916 & $10(13.70 \%)$ & $6(11.76 \%)$ & 0.752 \\
\hline Liver Cirrhosis (\%) & $94(41.78 \%)$ & $63(45.00 \%)$ & 0.546 & $48(65.75 \%)$ & $29(56.86 \%)$ & 0.315 \\
\hline Ascites (\%) & $105(46.67 \%)$ & $61(43.57 \%)$ & 0.564 & $40(54.79 \%)$ & $28(54.90 \%)$ & 0.991 \\
\hline Hepatic Encephalopathy (\%) & $6(2.67 \%)$ & $41(29.29 \%)$ & $<0.001$ & $2(2.74 \%)$ & $14(27.45 \%)$ & $<0.001$ \\
\hline Hepatic Encephalopathy I-II (\%) & $2(0.89 \%)$ & $25(17.86 \%)$ & & $2(2.74 \%)$ & $7(13.73 \%)$ & \\
\hline Hepatic Encephalopathy III-IV (\%) & $4(1.78 \%)$ & $16(11.43 \%)$ & & $0(0.00 \%)$ & $7(13.73 \%)$ & \\
\hline Infection (\%) & $43(19.11 \%)$ & $16(11.43 \%)$ & 0.055 & $14(19.18 \%)$ & $15(20.55 \%)$ & 0.185 \\
\hline Gastrointestinal hemorrhage (\%) & $7(3.11 \%)$ & $3(2.14 \%)$ & 0.584 & $4(5.48 \%)$ & $4(7.84 \%)$ & 0.557 \\
\hline sessions & $2.14 \pm 0.99$ & $1.94 \pm 1.04$ & 0.064 & $2.16 \pm 1.00$ & $2.04 \pm 1.11$ & 0.514 \\
\hline \multicolumn{7}{|l|}{ Laboratory parameters } \\
\hline $\operatorname{ALT}(\mathrm{U} / \mathrm{L})$ & $145.00(81.50,284.50)$ & $268.50(126.25,542.50)$ & $<0.001$ & $109.00(38.50,394.50)$ & $295.00(103.00,769.00)$ & 0.002 \\
\hline AST (U/L) & $120.00(84.00,208.00)$ & $176.50(104.25,336.00)$ & $<0.001$ & $113.00(57.00,335.50)$ & $240.00(95.00,491.00)$ & 0.069 \\
\hline TBil $(\mu \mathrm{mol} / \mathrm{L})$ & $404.12 \pm 112.48$ & $458.23 \pm 132.29$ & $<0.001$ & $376.25 \pm 118.29$ & $439.14 \pm 142.64$ & 0.009 \\
\hline ALB (g/L) & $32.12 \pm 7.64$ & $32.30 \pm 3.62$ & 0.792 & $33.58 \pm 4.96$ & $34.00 \pm 3.72$ & 0.595 \\
\hline GGT (U/L) & $66.00(47.00,86.50)$ & $75.50(56.00,101.00)$ & 0.011 & $78.00(46.50,128.50)$ & $68.00(41.00,108.00)$ & 0.439 \\
\hline Glucose $(\mathrm{mmol} / \mathrm{L})$ & $3.92(3.28,4.59)$ & $4.06(3.12,5.46)$ & 0.430 & $6.14(4.45,8.72)$ & $5.96(4.19,8.41)$ & 0.713 \\
\hline INR & $1.95(1.69,2.31)$ & $2.67(2.08,3.46)$ & $<0.001$ & $1.91(1.72,2.57)$ & $3.01(2.24,4.30)$ & $<0.001$ \\
\hline PT (s) & $21.70(18.85,25.40)$ & $30.55(23.05,37.88)$ & $<0.001$ & $21.60(19.10,27.20)$ & $32.50(22.70,45.70)$ & $<0.001$ \\
\hline Fibrinogen $(\mathrm{g} / \mathrm{L})$ & $1.31(1.04,1.61)$ & $1.08(0.87,1.40)$ & $<0.001$ & $1.42(1.07,1.83)$ & $1.20(0.74,1.46)$ & 0.002 \\
\hline $\mathrm{D}$-dimer $(\mu \mathrm{g} / \mathrm{L})$ & $2042.00(1012.50,3245.00)$ & $2586.50(1690.50,4359.50)$ & 0.001 & $972.00(434.00,2724.50)$ & $1998.00(1056.00,3132.00)$ & 0.013 \\
\hline $\operatorname{ALP}(\mathrm{U} / \mathrm{L})$ & $127.00(106.00,155.50)$ & $133.50(114.25,167.75)$ & 0.030 & $116.00(93.50,155.00)$ & $120.00(95.00,149.00)$ & 0.855 \\
\hline WBC $\left(10^{9} / \mathrm{L}\right)$ & $7.12 \pm 4.05$ & $9.52 \pm 3.84$ & $<0.001$ & $8.28 \pm 5.07$ & $10.26 \pm 5.71$ & 0.050 \\
\hline $\mathrm{Hb}(\mathrm{g} / \mathrm{L})$ & $122.53 \pm 18.91$ & $131.69 \pm 19.78$ & $<0.001$ & $109.96 \pm 26.49$ & $122.80 \pm 29.64$ & 0.015 \\
\hline Plt $\left(10^{9} / \mathrm{L}\right)$ & $108.23 \pm 47.98$ & $108.09 \pm 50.27$ & 0.979 & $105.52 \pm 59.52$ & $81.71 \pm 41.04$ & 0.015 \\
\hline Serum Sodium $(\mathrm{mmol} / \mathrm{L})$ & $137.00 \pm 4.17$ & $136.46 \pm 5.07$ & 0.284 & $137.96 \pm 5.08$ & $136.33 \pm 12.83$ & 0.394 \\
\hline Creatinine $(\mu \mathrm{mol} / \mathrm{L})$ & $67.00(59.00,80.00)$ & $66.00(57.25,83.50)$ & 0.732 & $60.00(49.50,92.50)$ & $69.00(41.00,100.00)$ & 0.739 \\
\hline GFR (ml/min) & $107.58(94.45,116.36)$ & $106.38(90.09,116.21)$ & 0.632 & $111.58(102.72,118.30)$ & $111.58(88.45,113.01)$ & 0.129 \\
\hline $\mathrm{BUN}(\mathrm{mmol} / \mathrm{L})$ & $4.00(3.25,5.40)$ & $5.00(3.30,6.73)$ & $<0.001$ & $4.70(3.35,9.24)$ & $5.61(3.41,8.30)$ & 0.631 \\
\hline $\mathrm{NH} 3(\mu \mathrm{mol} / \mathrm{L})$ & $65.00(48.00,84.50)$ & $73.00(53.75,110.25)$ & 0.002 & $69.00(46.00,90.00)$ & $103.00(71.10,170.00)$ & $<0.001$ \\
\hline HBV-DNA $(\log 10, \mathrm{IU} / \mathrm{ml})$ & $4.86 \pm 2.23$ & $5.32 \pm 2.20$ & 0.051 & $4.62 \pm 1.96$ & $4.60 \pm 1.60$ & 0.942 \\
\hline \multicolumn{7}{|l|}{ Scoring system } \\
\hline MELD score & $23.72 \pm 4.08$ & $28.09 \pm 6.49$ & $<0.001$ & $24.21 \pm 6.67$ & $28.98 \pm 7.87$ & $<0.001$ \\
\hline iMELD score & $41.76 \pm 6.42$ & $46.54 \pm 8.38$ & $<0.001$ & $40.85 \pm 9.53$ & $47.69 \pm 11.07$ & $<0.001$ \\
\hline CLIF-C ACLF score & $38.89 \pm 6.26$ & $44.83 \pm 6.58$ & $<0.001$ & $39.36 \pm 7.10$ & $45.51 \pm 6.27$ & $<0.001$ \\
\hline COSSH-ACLF score & $6.03(5.67,6.51)$ & $6.90(6.39,7.62)$ & $<0.001$ & $6.11(5.49,6.88)$ & $7.50(6.65,8.09)$ & $<0.001$ \\
\hline
\end{tabular}

Table 2. Baseline characteristics of derivation and validation cohorts, stratified by 28 day-mortality. Abbreviations: HTN, hypertension; DM, diabetes mellitus; ALT, alanine aminotransferase; AST, aspartate aminotransferase; TBil, total bilirubin; ALB, albumin; GGT, gamma-glutamyl transpeptidase; INR, internationalized normal ration; PT, prothrombin time; ALP, alkaline phosphatase; WBC, white blood cell; Hb, hemoglobin; Plt, platelet; GFR, glomerular filtration rate; BUN, urea nitrogen; HBV-DNA, hepatitis B virusdeoxyribonucleic acid; MELD, Model for End-stage Liver Disease; iMELD, integrated model for end-stage liver disease; CLIF-C ACLF, Chronic Liver Failure Consortium acute-on-chronic liver failure; COSSH-ACLF, Chinese group on the Study of Severe Hepatitis B-acute-on-chronic liver failure.

Helsinki and was approved by the Clinical Research Ethics Committees of the First Affiliated Hospital, Zhejiang University School of Medicine, and Shulan Hangzhou Hospital. Written informed consent was obtained from all patients or their legal surrogates prior to enrolment.

Inclusion and exclusion criteria. The enrolment criteria for the patients with HBV-ACLF corresponded to the COSSH-ACLF${ }^{4}$, which was developed to diagnose HBV-ACLF specifically. Briefly, HBV-ACLF was defined as acute deterioration of liver function and/or extrahepatic organ failure in patients underlying HBV-related chronic liver disease regardless of cirrhosis status. Detailed descriptions of the inclusion and exclusion criteria are given in the Supplemental Methods. 


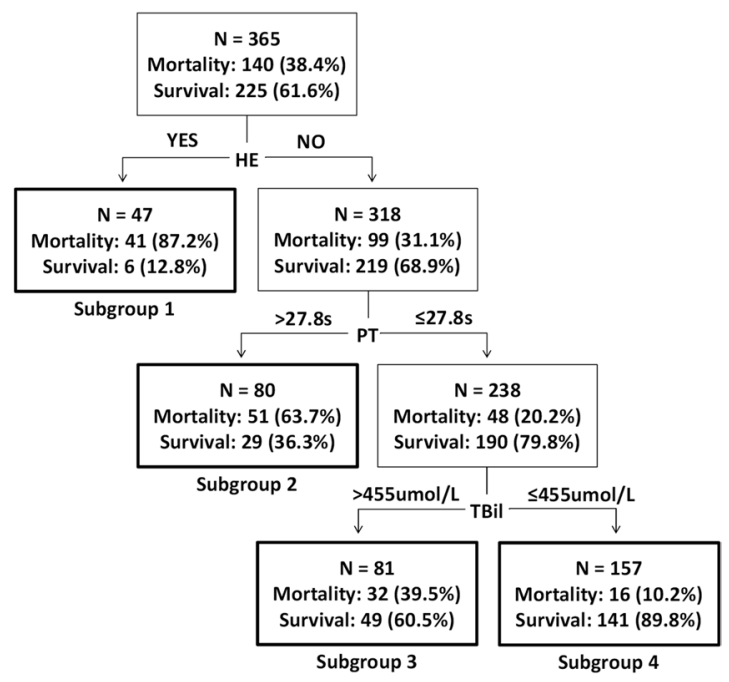

Figure 2. Predictors of ALSS therapy on HBV-ACLF patients and risk stratification for the derivation cohort.

\begin{tabular}{|c|c|c|c|c|c|c|c|c|}
\hline \multirow[b]{2}{*}{ Group } & \multicolumn{3}{|c|}{ Derivation cohort } & \multirow[b]{2}{*}{ P-value } & \multicolumn{3}{|c|}{ Validation cohort } & \multirow[b]{2}{*}{$P$-value } \\
\hline & \begin{tabular}{|l}
$\begin{array}{l}\text { No. of subjects } \\
(\%)\end{array}$ \\
\end{tabular} & Mortality (\%) & OR $(95 \% \mathrm{CI})$ & & \begin{tabular}{|l|}
$\begin{array}{l}\text { No. of subjects } \\
(\%)\end{array}$ \\
\end{tabular} & \begin{tabular}{|l} 
Mortality \\
$(\%)$
\end{tabular} & OR $(95 \% \mathrm{CI})$ & \\
\hline Low Risk & $238(65.21)$ & $50(21.00)$ & - & - & $74(59.68)$ & $16(21.62)$ & - & - \\
\hline High Risk & $127(34.79)$ & $92(72.44)$ & $9.883(6.002-16.275)$ & $<0.001$ & $50(40.32)$ & $35(70.00)$ & $8.485(3.726-19.202)$ & $<0.001$ \\
\hline Total & $365(100.00)$ & $142(38.90)$ & $2.394(1.644-3.488)$ & $<0.001$ & $124(100.00)$ & $51(41.13)$ & $2.533(1.310-4.895)$ & $<0.001$ \\
\hline
\end{tabular}

Table 3. 28-day mortality between risk groups.

Diagnostic criteria of complications. Liver cirrhosis was diagnosed based on symptoms and signs of portal hypertension and findings on ultrasonography, computed tomography or magnetic resonance imaging. Ascites was confirmed via paracentesis, abdominal imaging and other clinical evidence. HE assessment and grading employed the West Haven criteria ${ }^{33}$. Gastrointestinal haemorrhage was diagnosed by a positive faecal occult blood test or the presence of blood in vomit. Infections included spontaneous bacterial peritonitis, pulmonary infections and urinary tract infections, and were explored via imaging and laboratory culture ${ }^{34}$. Organ failure was diagnosed using the chronic liver failure-sequential organ failure assessment (CLIF-SOFA) score ${ }^{2}$.

Treatments. Standard medical therapy. All patients received standard medical therapy including bed rest, adequate nutritional support and single or combination of antiviral drugs. Sodium restriction, diuretics and paracentesis combined with albumin administration was used for ascites; Patients with hepatic encephalopathy received lactulose and L-ornithine aspartate; Appropriate antibiotics were applied for infections and adjusted based on the laboratory culture; Gastrointestinal haemorrhage were treated with somatostatin, pituitrin, proton pump inhibitors and necessary endoscopic therapy.

ALSS therapy. All patients received uniformed plasma exchange (PE) plus plasma bilirubin adsorption (PBA) ALSS therapy. For patients with HE, plasma perfusion (PP) was used as part of the ALSS therapy regimen ${ }^{10}$. The total exchanged plasma volume was $2500-3500 \mathrm{~mL}$, with the exchange rate was $20-25 \mathrm{~mL} / \mathrm{min}$. The flow rate of blood was $100-130 \mathrm{ml} / \mathrm{min}$. Dexamethasone $(5 \mathrm{mg})$ and heparin $(2500 \mathrm{U})$ were injected routinely before ALSS therapy. Protamine sulphate (20-50 mg) was used for neutralization in every session. Each session of ALSS therapy lasted for 4-6hours and was repeated every 2-4 days. ALSS therapy was discontinued when the overall improvement in the patient's status and $\mathrm{TB}<200 \mu \mathrm{mol} / \mathrm{L}$ or conditions such as bleeding and circulatory abnormalities that did not allow further ALSS therapy ${ }^{35}$. A total of 752 sessions (average 2 sessions/patient, ranging from 1 to 5 sessions) of ALSS therapy in derivation cohort, while a total of 262 sessions (average 2 sessions/ patient, ranging from 1 to 4 sessions) of ALSS therapy were performed in validation cohort (Supplementary Table 5).

ALSS therapy was initiated within 2 days of admission. The date of diagnosis of HBV-ACLF was the follow-up commencement date.

Data collection. We collected data on patient demographics and complications, laboratory measurements of alanine aminotransferase (ALT), aspartate aminotransferase (AST), TBil, albumin, gamma-glutamyl transpeptidase(GGT), sodium, and glucose; the INR; prothrombin time (PT); the levels of fibrinogen, D-dimer, alkaline phosphatase (ALP), and creatinine; the glomerular filtration rate (GFR); the levels of urea nitrogen (BUN), NH3, 


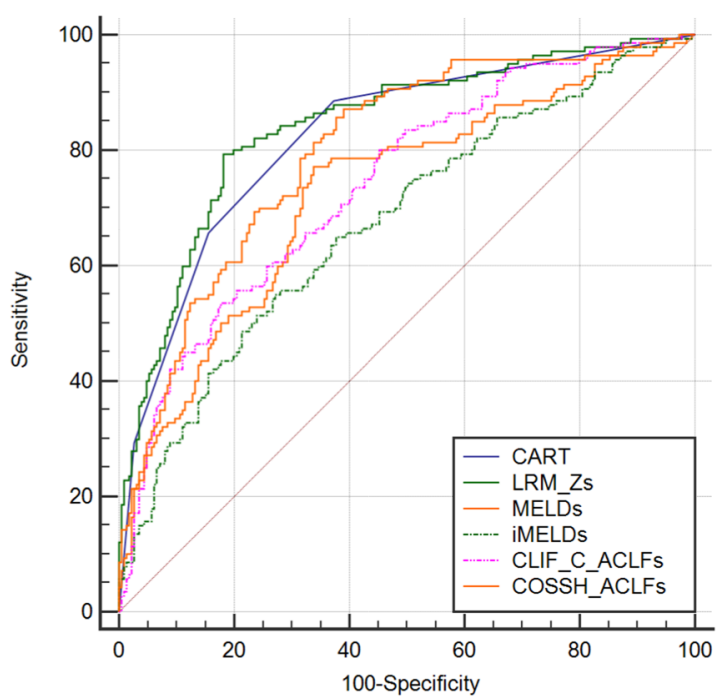

A

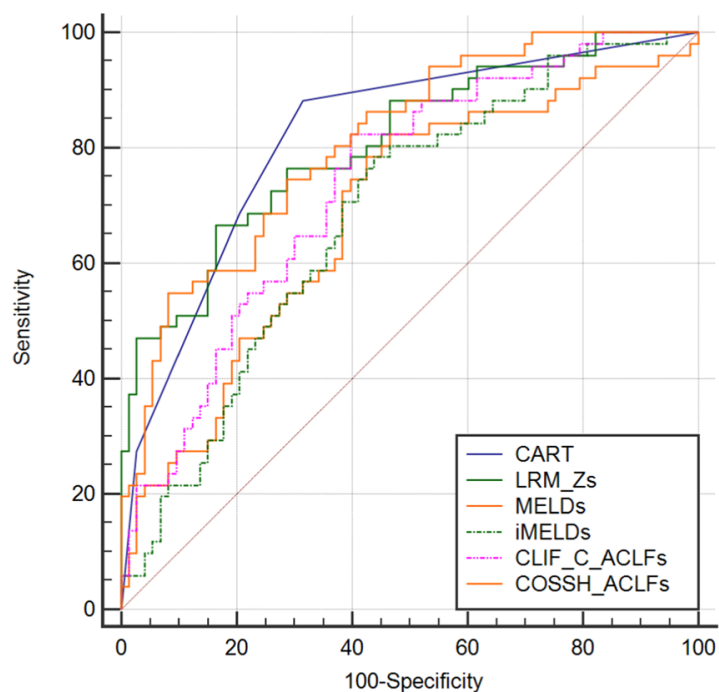

B

Figure 3. ROC analysis of the predictive accuracy of CART model, LRM-Z, MELD, iMELD, CLIF-C ACLF and COSSH-ACLF score to predict 28-day mortality of acute-on-chronic hepatitis B liver failure in derivation cohort (A) and validation cohort (B).

\begin{tabular}{|c|c|c|c|c|c|c|}
\hline \multirow[b]{2}{*}{ Models } & \multirow[b]{2}{*}{ auROC } & \multirow{2}{*}{\begin{tabular}{|l|}
$\begin{array}{l}\text { Derivation } \\
\text { cohort }\end{array}$ \\
$95 \%$ CI \\
\end{tabular}} & \multirow[b]{2}{*}{$P$-value } & \multirow[b]{2}{*}{ auROC } & \multirow{2}{*}{\begin{tabular}{|l|}
$\begin{array}{l}\text { Validation } \\
\text { cohort }\end{array}$ \\
$95 \%$ CI \\
\end{tabular}} & \multirow[b]{2}{*}{ P-valu } \\
\hline & & & & & & \\
\hline CART & 0.824 & $0.781-0.862$ & - & 0.820 & $0.741-0.883$ & - \\
\hline LRM-Z & 0.842 & $0.800-0.878$ & 0.1919 & 0.807 & $0.727-0.873$ & 0.6621 \\
\hline MELD & 0.727 & $0.678-0.772$ & 0.0001 & 0.686 & $0.597-0.766$ & 0.0070 \\
\hline iMELD & 0.675 & $0.625-0.723$ & $<0.0001$ & 0.685 & $0.596-0.766$ & 0.0152 \\
\hline CLIF-C ACLF & 0.742 & $0.694-0.786$ & 0.0011 & 0.738 & $0.651-0.812$ & 0.0985 \\
\hline COSSH-ACLF & 0.800 & $0.755-0.840$ & 0.1746 & 0.810 & $0.730-0.875$ & 0.7523 \\
\hline
\end{tabular}

Table 4. The predictive value of mortality of the CART score and other models in the derivation and validation cohorts. Abbreviations: CART, classification and regression tree; LRM-Z, logistic regression model Z; MELD, Model for End-stage Liver Disease; iMELD, integrated model for end-stage liver disease; CLIF-C ACLF,

Chronic Liver Failure Consortium acute-on-chronic liver failure; COSSH-ACLF, Chinese group on the Study of Severe Hepatitis B-acute-on-chronic liver failure. 
hepatitis B virus- deoxyribonucleic acid (HBV-DNA), white blood cell (WBC), hemoglobin (Hb) and platelet count (Plt). All laboratory data were collected at the time of hospital admission or prior to ALSS therapy.

The scores of published prognostic models (MELD, iMELD, CLIF-C ACLF and COSSH-ACLF) were calculated using the following formulas:

$$
\begin{aligned}
\text { MELD score }= & 9.6 \times \ln (\text { Creatinine }[\mathrm{mg} / \mathrm{dL}] / 88)+3.8 \times \ln (\mathrm{TBil}[\mathrm{umol} / \mathrm{L}] / 18) \\
+11.2 \times \ln (\mathrm{INR})+6.4 ; & \\
\text { iMELD score }=\text { MELD }+ & 0.3 \times \text { Age }[\text { years }]-0.7 \times \mathrm{Na}+[\mathrm{mmol} / \mathrm{L}]+100 \\
\text { CLIF }- \text { C ACLF score }= & 10 \times(0.33 \times \mathrm{CLIF}-\mathrm{OFs}+0.04 \times \text { Age }[\text { years }] \\
& \left.+0.63 \times \ln \left(\mathrm{WBC} \text { counts }\left[10^{9}\right]\right)-2\right) \\
\text { COSSH }- \text { ACLF score }= & 0.741 \times \mathrm{INR}+0.523 \times \mathrm{HBV}-\mathrm{SOFA} \\
& +0.026 \times \text { Age }[\text { years }]+0.003 \times \mathrm{TBil}[\mathrm{umol} / \mathrm{L}] .
\end{aligned}
$$

Construction of the CART and LRM-Z. Using selected variables, CART analysis divided all data into two homologous groups exhibiting different survival outcomes; the best splits and cut-off values were derived for each variable $e^{4,36-38}$. Then, the algorithm allocated data by reference to the best overall split of all best splits to a parent node, which then produce two child nodes exhibiting higher homogeneities. This process was repeated using both tree-building and -pruning until statistical analysis indicated that no further reduction in node impurity was possible or that pre-specified stop criteria had been met $^{39}$. This generated several subgroups with predicted mortality rates. We used CART analysis to identify and screen HBV-ACLF patients eligible for ALSS therapy. Two risk groups were identified base on the mortality rates of subgroups. The ability of CART to stratify ALSS-treated HBV-ACLF patients into subgroups by mortality risks was tested using the independent validation cohort. Patients from this cohort were allocated to subgroups using the flow chart of the CART tree. Mortality was calculated for each subgroup, and odds ratios (ORs) and 95\% confidence interval (CI) were calculated when risk groups were compared in both cohorts. Detailed descriptions of the LRM-Z construction are given in the Supplemental Methods.

Statistical analysis. Normality of distribution was explored for all variables. Continuous variables that were normally distributed were expressed as mean \pm standard deviations and other variables as median with interquartile ranges. Categorical variables are expressed as percentages and counts. Student's $t$-test or the MannWhitney U-test was used to compare continuous variables. The Pearson chi-squared test or Fisher's exact test was employed to compare categorical variables and proportions between groups, as appropriate. A $P$-value $<0.05$ was considered statistically significant. The capacities of various scoring systems to differentiate survivors from non-survivors were assessed by evaluating areas under receiver operating characteristic curves (auROCs). CART analysis was performed with the C50 package in R version 3.4 .3 (http://www.r-project.org/). Other statistical analyses was performed using SPSS ver. 18.0 software (SPSS Inc., Chicago, IL, USA). ROC curves were drawn with the aid of MedCalc ver. 18.2 software (Mariakerke, Belgium).

Received: 18 January 2019; Accepted: 28 October 2019;

Published online: 11 November 2019

\section{References}

1. Katoonizadeh, A. et al. Early features of acute-on-chronic alcoholic liver failure: a prospective cohort study. Gut 59, 1561-1569, https://doi.org/10.1136/gut.2009.189639 (2010).

2. Moreau, R. et al. Acute-on-chronic liver failure is a distinct syndrome that develops in patients with acute decompensation of cirrhosis. Gastroenterology 144, 1426-1437, 1437 e1421-1429, https://doi.org/10.1053/j.gastro.2013.02.042 (2013).

3. Sarin, S. K. et al. Acute-on-chronic liver failure: consensus recommendations of the Asian Pacific Association for the Study of the Liver (APASL) 2014. Hepatol Int 8, 453-471, https://doi.org/10.1007/s12072-014-9580-2 (2014).

4. Wu, T. et al. Development of diagnostic criteria and a prognostic score for hepatitis B virus-related acute-on-chronic liver failure. Gut, https://doi.org/10.1136/gutjnl-2017-314641 (2017).

5. Shen, Y. et al. Survival Benefits With Artificial Liver Support System for Acute-on-Chronic Liver Failure: A Time Series-Based MetaAnalysis. Medicine (Baltimore) 95, e2506, https://doi.org/10.1097/MD.0000000000002506 (2016).

6. Qin, G. et al. Artificial Liver Support System Improves Short- and Long-Term Outcomes of Patients With HBV-Associated Acuteon-Chronic Liver Failure. Medicine 93, e338, https://doi.org/10.1097/md.0000000000000338 (2014).

7. Hassanein, T. I., Schade, R. R. \& Hepburn, I. S. Acute-on-chronic liver failure: extracorporeal liver assist devices. Curr Opin Crit Care 17, 195-203, https://doi.org/10.1097/MCC.0b013e328344b3aa (2011).

8. Xu, X. et al. Artificial liver support system combined with liver transplantation in the treatment of patients with acute-on-chronic liver failure. PLoS One 8, e58738, https://doi.org/10.1371/journal.pone.0058738 (2013).

9. Heemann, U. et al. Albumin dialysis in cirrhosis with superimposed acute liver injury: a prospective, controlled study. Hepatology 36, 949-958, https://doi.org/10.1053/jhep.2002.36130 (2002).

10. Xia, Q. et al. A single-center experience of non-bioartificial liver support systems among Chinese patients with liver failure. Int $J$ Artif Organs 37, 442-454, https://doi.org/10.5301/ijao.5000341 (2014).

11. Ling, Q. et al. Downgrading MELD improves the outcomes after liver transplantation in patients with acute-on-chronic hepatitis B liver failure. PLoS One 7, e30322, https://doi.org/10.1371/journal.pone.0030322 (2012).

12. Kribben, A. et al. Effects of fractionated plasma separation and adsorption on survival in patients with acute-on-chronic liver failure. Gastroenterology 142, 782-789 e783, https://doi.org/10.1053/j.gastro.2011.12.056 (2012). 
13. Fonarow, G. C. et al. Risk stratification for in-hospital mortality in acutely decompensated heart failure: classification and regression tree analysis. JAMA 293, 572-580, https://doi.org/10.1001/jama.293.5.572 (2005).

14. Takahashi, O. et al. Risk stratification for in-hospital mortality in spontaneous intracerebral haemorrhage: a Classification and Regression Tree analysis. QJM 99, 743-750, https://doi.org/10.1093/qjmed/hcl107 (2006).

15. Shi, K. Q. et al. Classification and regression tree analysis of acute-on-chronic hepatitis B liver failure: Seeing the forest for the trees. J Viral Hepat 24, 132-140, https://doi.org/10.1111/jvh.12617 (2017).

16. Zhao, R. H., Shi, Y., Zhao, H., Wu, W. \& Sheng, J. F. Acute-on-chronic liver failure in chronic hepatitis B: an update. Expert Rev Gastroenterol Hepatol 12, 341-350, https://doi.org/10.1080/17474124.2018.1426459 (2018).

17. Shen, Y. et al. A comprehensive validation of HBV-related acute-on-chronic liver failure models to assist decision-making in targeted therapeutics. Sci Rep 6, 33389, https://doi.org/10.1038/srep33389 (2016).

18. Gustot, T. \& Agarwal, B. Selected patients with acute-on-chronic liver failure grade 3 are not too sick to be considered for liver transplantation. J Hepatol 67, 667-668, https://doi.org/10.1016/j.jhep.2017.07.017 (2017).

19. Artru, F. et al. Liver transplantation in the most severely ill cirrhotic patients: A multicenter study in acute-on-chronic liver failure grade 3. J Hepatol 67, 708-715, https://doi.org/10.1016/j.jhep.2017.06.009 (2017).

20. Nian, X. et al. Association between hepatitis B virus basal core promoter/precore region mutations and the risk of hepatitis B-related acute-on-chronic liver failure in the Chinese population: an updated meta-analysis. Hepatol Int 10, 606-615, https://doi.org/10.1007/ s12072-016-9716-7 (2016).

21. Qin, G. et al. Logistic regression model can reduce unnecessary artificial liver support in hepatitis B virus-associated acute-onchronic liver failure: decision curve analysis. BMC Med Inform Decis Mak 16, 59, https://doi.org/10.1186/s12911-016-0302-7 (2016).

22. Cordoba, J. et al. Characteristics, risk factors, and mortality of cirrhotic patients hospitalized for hepatic encephalopathy with and without acute-on-chronic liver failure (ACLF). J Hepatol 60, 275-281, https://doi.org/10.1016/j.jhep.2013.10.004 (2014).

23. Romero-Gomez, M., Montagnese, S. \& Jalan, R. Hepatic encephalopathy in patients with acute decompensation of cirrhosis and acute-on-chronic liver failure. J Hepatol 62, 437-447, https://doi.org/10.1016/j.jhep.2014.09.005 (2015).

24. Garg, H. et al. Clinical profile and predictors of mortality in patients of acute-on-chronic liver failure. Dig Liver Dis 44, 166-171, https://doi.org/10.1016/j.dld.2011.08.029 (2012).

25. Jalan, R. et al. Acute-on chronic liver failure. J Hepatol 57, 1336-1348, https://doi.org/10.1016/j.jhep.2012.06.026 (2012).

26. Bao, S. et al. Role of interleukin-23 in monocyte-derived dendritic cells of HBV-related acute-on-chronic liver failure and its correlation with the severity of liver damage. Clin Res Hepatol Gastroenterol 41, 147-155, https://doi.org/10.1016/j.clinre.2016.10.005 (2017).

27. Ren, Y. et al. Development and validation of a scoring system to predict progression to acute-on-chronic liver failure in patients with acute exacerbation of chronic hepatitis B. Hepatol Res 48, 692-700, https://doi.org/10.1111/hepr.13062 (2018).

28. Choudhury, A. et al. Liver failure determines the outcome in patients of acute-on-chronic liver failure (ACLF): comparison of APASL ACLF research consortium (AARC) and CLIF-SOFA models. Hepatol Int 11, 461-471, https://doi.org/10.1007/s12072-0179816-z (2017).

29. Gao, F. et al. Development and validation of a prognostic model for acute-on-chronic hepatitis B liver failure. Eur J Gastroenterol Hepatol 29, 669-678, https://doi.org/10.1097/MEG.0000000000000854 (2017).

30. Chen, X., Wang, M. \& Zhang, H. The use of classification trees for bioinformatics. Wiley Interdiscip Rev Data Min Knowl Discov 1, 55-63, https://doi.org/10.1002/widm.14 (2011).

31. Speybroeck, N. Classification and regression trees. Int J Public Health 57, 243-246, https://doi.org/10.1007/s00038-011-0315-z (2012).

32. Lemon, S. C., Roy, J., Clark, M. A., Friedmann, P. D. \& Rakowski, W. Classification and regression tree analysis in public health: methodological review and comparison with logistic regression. Ann Behav Med 26, 172-181, https://doi.org/10.1207/ S15324796ABM2603_02 (2003).

33. Blei, A. T. \& Cordoba, J., Practice Parameters Committee of the American College of, G. Hepatic Encephalopathy. Am J Gastroenterol 96, 1968-1976, https://doi.org/10.1111/j.1572-0241.2001.03964.x (2001).

34. Yin, S. et al. Risk of different precipitating events for progressing to acute-on-chronic liver failure in HBV-related cirrhotic patients. J Dig Dis 18, 292-301, https://doi.org/10.1111/1751-2980.12480 (2017).

35. Jia-Jia Chen et al. Plasma exchange-centered artificial liver support system in hepatitis B virus-related acute-on-chronic liver failure: a nationwide prospective multicenter study in China. Hepatobiliary \& Pancreatic Diseases International 15, 275-281, https://doi. org/10.1016/S1499-3872(16)60084-X (2016).

36. Malinchoc, M. et al. A model to predict poor survival in patients undergoing transjugular intrahepatic portosystemic shunts. Hepatology 31, 864-871, https://doi.org/10.1053/he.2000.5852 (2000).

37. Luca, A. et al. An integrated MELD model including serum sodium and age improves the prediction of early mortality in patients with cirrhosis. Liver Transpl 13, 1174-1180, https://doi.org/10.1002/lt.21197 (2007).

38. Jalan, R. et al. Development and validation of a prognostic score to predict mortality in patients with acute-on-chronic liver failure. J Hepatol 61, 1038-1047, https://doi.org/10.1016/j.jhep.2014.06.012 (2014).

39. Marshall, R. J. The use of classification and regression trees in clinical epidemiology. J Clin Epidemiol 54, 603-609 (2001).

\section{Acknowledgements}

This study was supported by Chinese High Tech Research and Development (863) Program (2012AA020204, 2013AA020102), The National S\&T Major Project (2012ZX10002004-001), The National Key Research and Development Program of China (2016YFC1101304/3) and Key research and development project of Department of Science and Technology of Zhejiang Province (2017C03051).

\section{Author contributions}

Kaizhou Huang, Feiyang Ji and Zhongyang Xie contributed to this paper equally. Kaizhou Huang co-conceived the research, collected clinical information, performed the LRM analysis, prepared the figures and tables and drafted the manuscript. Feiyang Ji co-conceived the research and performed the CART analysis. Zhongyang Xie co-conceived the research, collected clinical information and drafted the manuscript. Daxian Wu, Xiaowei $\mathrm{Xu}$ and Hainv Gao reviewed and analyzed the clinical data. Xiaoxi Ouyang, Lanlan Xiao, Menghao Zhou and Danhua Zhu collected clinical information. All authors reviewed the manuscript.

\section{Competing interests}

The authors declare no competing interests.

Additional information

Supplementary information is available for this paper at https://doi.org/10.1038/s41598-019-53029-0. 
Correspondence and requests for materials should be addressed to L.L.

Reprints and permissions information is available at www.nature.com/reprints.

Publisher's note Springer Nature remains neutral with regard to jurisdictional claims in published maps and institutional affiliations.

(1) Open Access This article is licensed under a Creative Commons Attribution 4.0 International License, which permits use, sharing, adaptation, distribution and reproduction in any medium or format, as long as you give appropriate credit to the original author(s) and the source, provide a link to the Creative Commons license, and indicate if changes were made. The images or other third party material in this article are included in the article's Creative Commons license, unless indicated otherwise in a credit line to the material. If material is not included in the article's Creative Commons license and your intended use is not permitted by statutory regulation or exceeds the permitted use, you will need to obtain permission directly from the copyright holder. To view a copy of this license, visit http://creativecommons.org/licenses/by/4.0/.

(C) The Author(s) 2019 